\title{
Blackout Avoidance through Intelligent Load Shedding in Modern Electrical Power Utility Network
}

\author{
Ishtiaq Ahmad', Faizullah Khan², Surat Khan², Akbar Khan³, Abdul Wahid Tareen², Muhammad Saeed
}

\begin{abstract}
${ }^{1}$ Department of Electrical Engineering, ${ }^{2}$ Department of Telecommunication Engineering, ${ }^{3}$ Department of Computer Engineering, Faculty of Information and Communication Technology, Balochistan University of Information Technology, Engineering and Management Sciences, Quetta, Pakistan, ${ }^{4}$ Department of Electrical Engineering, National University of Computer and Emerging Sciences Islamabad, Pakistan
\end{abstract}

\begin{abstract}
During the last few decades many blackouts have occurred throughout the world. It seems that modern power systems are more vulnerable to major blackouts. Power system in Pakistan is no exception where blackouts affect the economy and hinder the development of the country. Pakistan Electric Power Company (PEPCO) is responsible for generation, transmission, and distribution of electric power in Pakistan. Major blackouts occur due to failure of the protection system of the power transmission network. The remedy for such collapses is disconnection of non-preferred load through Intelligent Load Shedding (ILS) technique. In this paper, the Intelligent Load Shedding is simulated in Electrical Transient Analysis Program (ETAP) software. The technique has been applied on 220kV transmission network of Quetta Electric Supply Company (QESCO), a power distribution company in Balochistan province of Pakistan. This paper focuses on preventing entire QESCO network from cascaded tripping and blackout during $\mathrm{N}-2$ contingency situations. It has been established that implementation of Intelligent Load Shedding is not only helpful for protecting the transmission network of the distribution company, like QESCO, from blackout, but also protects smart grid from blackouts and other power collapses.
\end{abstract}

Keywords: Power System; Blackout; Cascaded Tripping; Intelligent Load Shedding; N-2 Contingency

Corresponding author's email: engr.ishtiaqmarwat@gmail.com

\section{INTRODUCTION}

Electrical power nowadays is considered as the life line for any society. Unavailability of uninterrupted electric power results in failure of public transport system, traffics jams, educational institution outages, breakdown of industries and so on. Electrical power outages literally cripple the economy of a country.

The European Network of Transmission System Operators for Electricity defined blackout as "the interruption of electricity generation, transmission, distribution and consumption processes, when operation of the transmission system or a part thereof is terminated". Power blackout is a complicated phenomenon and occurs due to unusual events in a power network (Adibi, 2015) The initiators of power system blackout have been generalized to atmospheric phenomena, technical causes and human factors (Złotecka and Sroka, 2018). The mechanisms of large scale power blackouts can be analysed through power failure models (Wang et al., 2018). Traditional methods have been used to prevent blackout. Merz and Mc Lellan proposed that in a power blackout situation, power protection system plays a crucial role. Protection system prompts swift isolation of the faulty component, thereby avoiding blackout through a smart protection system (McLellzm, 2010). M. El-werfelli et al. analysed the causes of Libyan blackout and concluded that improper protection of the generating units led to the power network blackout. He also suggested that there should be proper co-ordination between the protection systems of the generating units and power grid. Erica Fong introduced "Alert Management System" to control the energy demand by very large number of consumers that could cause potential blackout. The Alert management system supervises the serious energy intake events for avoiding the system outage. The system could be installed at the supplier's side to prevent energy usage interruption and blackout (Fong et al., 2011). Adeniyi A. Babalola used the technique of an adaptive multi-agent system algorithm to prevent cascading failure (Babalola and Belkacemi, 2018).

The power sector of Pakistan faces frequent blackouts. A lot of work has been done for the blackout avoidance. Younas and Qureshi investigated in 2006 National Grid blackout and suggested the installation 
of power system controllers like Power System Stabilizers (PSS) and Flexible AC Transmission Systems (FACTS) (Younas and Qureshi, 2006). For highly stressed grids, Noman Bashir and Zohaib Sherani introduced Ashiyana, an improved and practical form of Direct Load Control (DLC). It was better than manual load-shedding systems as it could decrease the number of customer with no power by $80 \%$. This technique also prevented the utility companies from facing social unrest as well as power security to consumers (Bashir et al., 2015). Blackouts started in Pakistan after 1980. The network of National Grid is connected in an inter-connected system (Kafait-Ullah, 2013).

The main focus of this research work is to avoid blackout in Quetta Electric Supply Company (QESCO) region of Pakistan and to improve the quality of service of the National Grid. In this research work idea of Intelligent Load Shedding (ILS) system has been presented. Through ILS non-preferred load of a power system can be shed at any time. The concept of intelligent load shedding is simulated through Electrical Transient Analysis Program (ETAP).

Table 1 shows various blackouts that occurred in different countries of the world due to transmission lines faults (Scutariu and MacDonald, 2009).

Table 1: Blackouts due to line fault

\begin{tabular}{|l|l|c|c|l|}
\hline Date & Country & $\begin{array}{c}\text { Power Loss } \\
\text { (GW) }\end{array}$ & $\begin{array}{c}\text { Population Affected } \\
\text { (million) }\end{array}$ & Blackout Cause \\
\hline Jan-2007 & Croatia & 1.30 & 2 & Numerous overloads \\
\hline Aug2003 & USA & 6.1 & 50 & High voltage drops \\
\hline Sep2003 & Denmark & 6.6 & 4 & $\begin{array}{l}\text { Islanded system } \\
\text { breakdown }\end{array}$ \\
\hline Sep2003 & Italy & 0.24 & 57 & Loss of synchronism \\
\hline Nov2006 & Poland & 1.7 & 15 & Line overloads \\
\hline Jan-2016 & Pakistan & 0.12 & 56 & 220kV Towers damage \\
\hline
\end{tabular}

\section{MATERIALS AND METHODS}

\section{Implementation of ILS through ETAP}

Intelligent Load Shedding (ILS) is the process of optimal and fast disconnection of non-preferred load from a power system to keep the rest of the system operational (Yagami and Ichinohe, 2017). Intelligent Load Shedding is sometimes called automatic load shedding. The purpose of this load reduction is to protect the whole power system or power utility network from large disturbances and power outages.

\section{0 kV Transmission System of QESCO}

Quetta Electric Supply Company (QESCO) is responsible for supplying electrical power throughout Balochistan province of Pakistan except district Lasbela which is fed by K-Electric Karachi. QESCO has the power network of three $220 \mathrm{kV}$ transmission lines coming from different locations. Following is the detail of 220kV transmission lines of QESCO network:

1. 220kV Daddu (Sindh)-Khuzdar (Balochistan) region

2. 220kV DG-Khan (Punjab)-Loralai (Balochistan) region

3. 220kV Guddu (Sindh) - Sibbi (Balochistan) region

The total $132 \mathrm{kV}$ transmission lines and grid stations fed by above $220 \mathrm{kV}$ transmission lines are listed in Table-2.

We can convert this load (ampere) into three phase real power (MW) and apparent power (MVA) if we have the voltage and power factor of the load. We have;

$$
\begin{aligned}
& \text { Where, } \\
& P=\text { Real Power (MW) } \\
& V=\text { Voltage across load }(\mathrm{v}) \\
& I=\text { Load Current }(\mathrm{A}) \\
& \cos \theta=\text { Load Power Factor }
\end{aligned}
$$

$$
P=\sqrt{3} V I \cos \theta
$$


The Single Line Diagram (SLD) of all connected loads, transformers and transmission lines are given in Figure-1.

Table 2: Maximum Connected Load of QESCO Region

\begin{tabular}{|c|c|c|c|c|c|c|}
\hline S.No. & Name of Grid Station & Transformers Connected & $\begin{array}{l}\text { Maximum Load } \\
\text { (A) }\end{array}$ & $\begin{array}{l}\text { Maximum Load } \\
\text { (MVA) }\end{array}$ & $\begin{array}{l}\text { Maximum Load } \\
\text { (NIV) }\end{array}$ & Date \\
\hline \multirow[b]{2}{*}{1} & \multirow[b]{2}{*}{$132 \mathrm{kV} \mathrm{G} \mathrm{S} \mathrm{Sariab}$} & T-I (40MVA) & 1275 & 25.4 & 20 & $20-04-2017$ \\
\hline & & T-II (40MVA) & 1640 & 32.7 & 26 & $20-04-2017$ \\
\hline \multirow[b]{2}{*}{2} & \multirow[b]{2}{*}{$132 \mathrm{kV}$ GS Marriabad } & T-I (26MVA) & 1010 & 20 & 16 & $18-04-2017$ \\
\hline & & T-II (26MVA) & 490 & 9.8 & 7.8 & $18-04-2017$ \\
\hline \multirow[b]{2}{*}{3} & \multirow[b]{2}{*}{ 132kV G S Quetta City } & T-I (40MVA) & 1483 & 29.5 & 23.6 & $27-04-2017$ \\
\hline & & T-II (40MVA) & 1500 & 30 & 24 & $27-04-2017$ \\
\hline \multirow[b]{2}{*}{4} & \multirow[b]{2}{*}{$132 \mathrm{kV}$ G S Shiekhmanda } & $\mathrm{T}-\mathrm{I}(40 \mathrm{MVA})$ & 1460 & 29 & 23.3 & $21-04-2017$ \\
\hline & & T-II (40MVA) & 1490 & 30 & 24 & 21-04-2017 \\
\hline \multirow{4}{*}{5} & \multirow{4}{*}{$132 \mathrm{kV}$ G S Pishin } & T-I (40MVA) & 1490 & 30 & 24 & $08-04-2017$ \\
\hline & & T-II (40MVA) & 1760 & 35 & 28 & $08-04-2017$ \\
\hline & & T-III (6.3MVA) & 76 & 1.5 & 1.2 & $08-04-2017$ \\
\hline & & T-IV (40MVA) & 1280 & 25.5 & 20.4 & $08-04-2017$ \\
\hline \multirow[t]{3}{*}{6} & \multirow{3}{*}{$132 \mathrm{kV} \mathrm{G} \mathrm{S} \mathrm{Q} \mathrm{Abdullah}$} & T-I (40MVA) & 1280 & 25.5 & 20.4 & $18-04-2017$ \\
\hline & & T-II (26MVA) & 1280 & 25.5 & 20.4 & $18-04-2017$ \\
\hline & & T-III (26MVA) & 1280 & 25.5 & 20.4 & 18-04-2017 \\
\hline 7 & 132kV G S Sorrange & T-I (7.5MVA) & 246 & 5 & 4 & $12-04-2017$ \\
\hline \multirow[t]{2}{*}{8} & $132 \mathrm{kV} \mathrm{GS} \mathrm{Yaru}$ & T-I (26MVA) & 1320 & 26.3 & 21 & $10-04-2017$ \\
\hline & & T-II (26MVA) & 1050 & 21 & 16.7 & $10-04-2017$ \\
\hline 9 & 132kV GS Alizai & T-I (26MVA) & 940 & 18.7 & 15 & $21-04-2017$ \\
\hline & & T-ПI (26MVA) & 885 & 17.6 & 14 & $21-04-2017$ \\
\hline 10 & 132kV G S Huramzai & T-I (26MVA) & 1280 & 25.5 & 20.4 & $22-04-2017$ \\
\hline 11 & 132kV GS Chaman & T-I (13MVA) & 972 & 19.4 & 15.5 & 23-04-2017 \\
\hline & & T-П (26MVA) & 990 & 19.7 & 15.8 & $23-04-2017$ \\
\hline 12 & 132kV G S Kuchlak & T-I (26MVA) & 741 & 14.76 & 11.8 & $21-04-2017$ \\
\hline & & T-I (40MVA) & 1571 & 31.3 & 25 & $16-04-2017$ \\
\hline 13 & $132 \mathrm{kV} \mathrm{G}$ S Gulistan & T-II (26MVA) & 1574 & 31.4 & 25 & 16-04-2017 \\
\hline & & T-III (26MVA) & 1242 & 25 & 20 & $16-04-2017$ \\
\hline 14 & 132kV G S Sharig & T-I (6.3MVA) & 260 & 5.2 & 4 & $08-04-2017$ \\
\hline 15 & $132 \mathrm{kV} \mathrm{G} \mathrm{S} \mathrm{Hernai}$ & T-I (6.3MVA) & 274 & 5.5 & 4.4 & $13-04-2017$ \\
\hline 16 & $132 \mathrm{kV} \mathrm{GS} \mathrm{Mach}$ & T-I (6.3MVA) & 250 & 5 & 4 & $26-04-2017$ \\
\hline 17 & $132 \mathrm{kV}$ GiS Bagh & T-I (26MVA) & 910 & 18 & 14.5 & $19-04-2017$ \\
\hline 18 & 132kV G S Darwaza & T-I (26MVA) & 1180 & 37 & 30 & $26-04-2017$ \\
\hline & & T-1 (26MVA) & 1180 & 37 & 30 & $15-04-2017$ \\
\hline 19 & $132 \mathrm{kV}$ G S Khanak & T-II (26MVA) & 800 & 16 & 12.8 & $15-04-2017$ \\
\hline & & T-I (26MVA) & 1150 & 23 & 18 & $16-04-2017$ \\
\hline 20 & $132 \mathrm{kV} \mathrm{G} \mathrm{SSibbi}$ & T-II (26MVA) & 1100 & 22 & 17.5 & $16-0+-2017$ \\
\hline & & T-I (26MVA) & 1096 & 21.8 & 17.5 & $12-04-2017$ \\
\hline 21 & $132 \mathrm{kV} \mathrm{G} \mathrm{S} \mathrm{Loralai}$ & T-II (40MVA) & 1885 & 37.5 & 30 & $12-04-2017$ \\
\hline & & T-III (6.3MVA) & 0 & 0 & 0 & $12-04-2017$ \\
\hline 22 & 132kV G S Makhter & T-I (13MVA) & 393 & 7.8 & 6.2 & 15-04-2017 \\
\hline 23 & 132kV G S Kingri & T-I (6.3MVA & 266 & 5.3 & 4.2 & $15-04-2017$ \\
\hline & & T-I (13MVA) & 440 & 8.8 & 7 & 21-04-2017 \\
\hline 24 & 132kV G S Rakhni & T-II (26MVA) & 860 & 17 & 13.7 & $21-04-2017$ \\
\hline & & T-I (26MVA) & 698 & 14 & 11 & $20-04-2017$ \\
\hline 25 & 132kV G S Barkhan & T-II (13MVA) & 550 & 11 & 8.8 & $20-04-2017$ \\
\hline 26 & 132kV G S Kohlu & T-I (13MVA) & 601 & 12 & 9.6 & 27-04-2017 \\
\hline & & T-I (13MVA) & 475 & 9.5 & 7.6 & $03-04-2017$ \\
\hline 27 & $132 \mathrm{kV} \mathrm{GS} \mathrm{Zhob}$ & T-П (26MVA) & 955 & 19 & 15.2 & $03-04-2017$ \\
\hline & & T-I (40MVA) & 995 & 19.8 & 15.8 & $09-04-2017$ \\
\hline 28 & $132 \mathrm{kV} \mathrm{G} \mathrm{S} \mathrm{Q} \mathrm{Saifullah}$ & T-II (40MVA) & 1030 & 20.5 & 16.4 & $09-04-2017$ \\
\hline & & T-III (40MVA) & 990 & 19.7 & 15.8 & $09-04-2017$ \\
\hline 29 & 132kV GS M. Bagh & T-I (26MVA) & 1070 & 21.3 & 17 & $25-04-2017$ \\
\hline & & T-II (26MVA) & 860 & 17 & 13.7 & $25-04-2017$ \\
\hline 30 & 132kV G.S G.H Zai & T-I (26MVA) & 1100 & 22 & 17.5 & $07-04-2017$ \\
\hline & & T-II (13MVA) & 170 & 3.4 & 2.7 & $07-04-2017$ \\
\hline 31 & $132 \mathrm{kV} \mathrm{G} \mathrm{S} \mathrm{M} \mathrm{Pur}$ & T-I (6.3MVA) & 230 & 4.6 & 3.7 & $03-04-2017$ \\
\hline & & T-II (26MVA) & 430 & 8.6 & 6.8 & $03-04-2017$ \\
\hline 32 & 132kV G S Khanozai & T-I (26MVA) & 1163 & 23 & 18.5 & $28-04-2017$ \\
\hline & & T-II (26MVA) & 1194 & 24 & 19 & $28-04-2017$ \\
\hline 33 & $132 \mathrm{kV} \mathrm{G/S} \mathrm{Ziarat}$ & T-I (26MVA) & 670 & 13,3 & 10.7 & $28-04-2017$ \\
\hline & & T-I (13MVA) & 990 & 19.7 & 15.8 & $12-04-2017$ \\
\hline 34 & $132 \mathrm{kV} \mathrm{G} \mathrm{S} \mathrm{Dukki}$ & T-II (13MVA) & 370 & 7.4 & 6 & $12-04-2017$ \\
\hline & & T-I (26 MVA $)$ & 1110 & 22 & 17.7 & $08-04-2017$ \\
\hline 35 & $132 \mathrm{kv}$ G S Mastung & T-II (26 MVA) & 906 & 18 & 14.4 & $08-04-2017$ \\
\hline & & T-III (26 MVA) & 920 & 18.3 & 14.7 & $08-04-2017$ \\
\hline
\end{tabular}


Blackout Avoidance through Intelligent Load Shedding in Modern Electrical Power Utility Network

\begin{tabular}{|c|c|c|c|c|c|c|}
\hline \multirow[b]{2}{*}{36} & \multirow[b]{2}{*}{ 132kv G/S Panjpai } & T-I (26 MVA) & 700 & 14 & 11 & 14-04-2017 \\
\hline & & T-II (13MVA) & 440 & 8.8 & 7 & $15-04-2017$ \\
\hline \multirow[b]{2}{*}{37} & \multirow[b]{2}{*}{ 132kv G/S Kirdgab } & T-I (26 MVA) & 1010 & 20 & 16 & $17-04-2017$ \\
\hline & & T-II (13MVA) & 500 & 10 & 8 & $21-04-2017$ \\
\hline 38 & 132kv G/S Noshki & T-II (26 MVA) & 1282 & 25.5 & 20.4 & $23-04-2017$ \\
\hline \multirow{3}{*}{39} & \multirow{3}{*}{$132 \mathrm{kv}$ G/S Kharan } & T-I (26 MVA) & 880 & 17.5 & 14 & $20-04-2017$ \\
\hline & & T-II (26 MVA) & 820 & 16.3 & 13 & 20-04-2017 \\
\hline & & T-III (26 MVA) & 1160 & 23 & 18.5 & 20-04-2017 \\
\hline \multirow[b]{2}{*}{40} & \multirow[b]{2}{*}{ 132kv G/S Khad Kucha } & T-I (26 MVA) & 1170 & 23.3 & 18.6 & $10-04-2017$ \\
\hline & & T-II (26 MVA) & 1170 & 23.3 & 18.6 & $13-04-2017$ \\
\hline \multirow{3}{*}{41} & \multirow{3}{*}{ 132kv G/S Mangochar } & T-I (40MVA) & 1920 & 38.2 & 30.6 & 28-04-2017 \\
\hline & & T-II (26 MVA) & 1100 & 22 & 17.7 & $28-04-2017$ \\
\hline & & T-III (26 MVA) & 1120 & 22.3 & 17.8 & $28-04-2017$ \\
\hline \multirow{3}{*}{42} & \multirow{3}{*}{$132 \mathrm{kv}$ G/S Kalat } & T-I (26 MVA) & 1270 & 25 & 20 & $14-04-2107$ \\
\hline & & T-II (40 MVA) & 980 & 19.5 & 15.6 & 14-04-2107 \\
\hline & & T-III (26 MVA) & 800 & 16 & 12.7 & $14-04-2107$ \\
\hline 43 & $132 \mathrm{kv}$ G/S Surab & T-II (13MVA) & 650 & 13 & 10.4 & $07-04-2017$ \\
\hline \multirow[b]{2}{*}{44} & \multirow[b]{2}{*}{ 132kV G/S Gidder } & T-I (40 MVA) & 1060 & 21 & 17 & 25-04-2017 \\
\hline & & T-II (13MVA) & 630 & 12.6 & 10 & $25-04-2017$ \\
\hline \multirow[b]{2}{*}{45} & \multirow[b]{2}{*}{ 132kV G S Bagh Bana } & T-I (40 MVA) & 1300 & 26 & 20.7 & 13-04-2017 \\
\hline & & T-II (26 MVA) & 590 & 11.8 & 9.4 & $13-04-2017$ \\
\hline \multirow{3}{*}{46} & \multirow{3}{*}{ 132kV G/S Khuzdar } & T-I (40 MVA) & 1570 & 31.3 & 25 & $07-04-2017$ \\
\hline & & T-II (18MVA) & 700 & 14 & 11 & $07-04-2017$ \\
\hline & & T-III (26 MVA) & 1060 & 21 & 17 & $07-04-2017$ \\
\hline \multirow[b]{2}{*}{47} & \multirow[b]{2}{*}{$132 \mathrm{kV} \mathrm{G} / \mathrm{S} \mathrm{Nal}$} & T-I (26 MVA) & 1150 & 23 & 18 & $03-04-2017$ \\
\hline & & T-II (26 MVA) & 1290 & 25.7 & 20.6 & $03-04-2017$ \\
\hline \multirow{3}{*}{48} & \multirow{3}{*}{$132 \mathrm{kV} \mathrm{G}$ S Wadh } & T-I (26 MVA) & 1126 & 22.4 & 18 & $07-04-2017$ \\
\hline & & T-II (13MVA) & 617 & 12.3 & 9.8 & $07-04-2017$ \\
\hline & & T-III (13MVA) & 390 & 7.8 & 6.2 & $07-04-2017$ \\
\hline \multirow[t]{2}{*}{49} & 132kV G/S Zehri & T-I (26 MVA) & 1110 & 22 & 17.7 & $10-04-2017$ \\
\hline & & T-II (13MVA) & 360 & 7.2 & 5.7 & $10-04-2017$ \\
\hline 50 & 132kV G/S Dalbandin & T-I (26 MVA) & 150 & 3 & 2.4 & 22-04-2017 \\
\hline 53 & 132kV G S Baseema & T-I (13MVA) & 221 & 4.4 & 3.5 & 23-04-2017 \\
\hline TOT & LOAD CONNECTED & & 94592 & 1912 & 1527 & \\
\hline
\end{tabular}




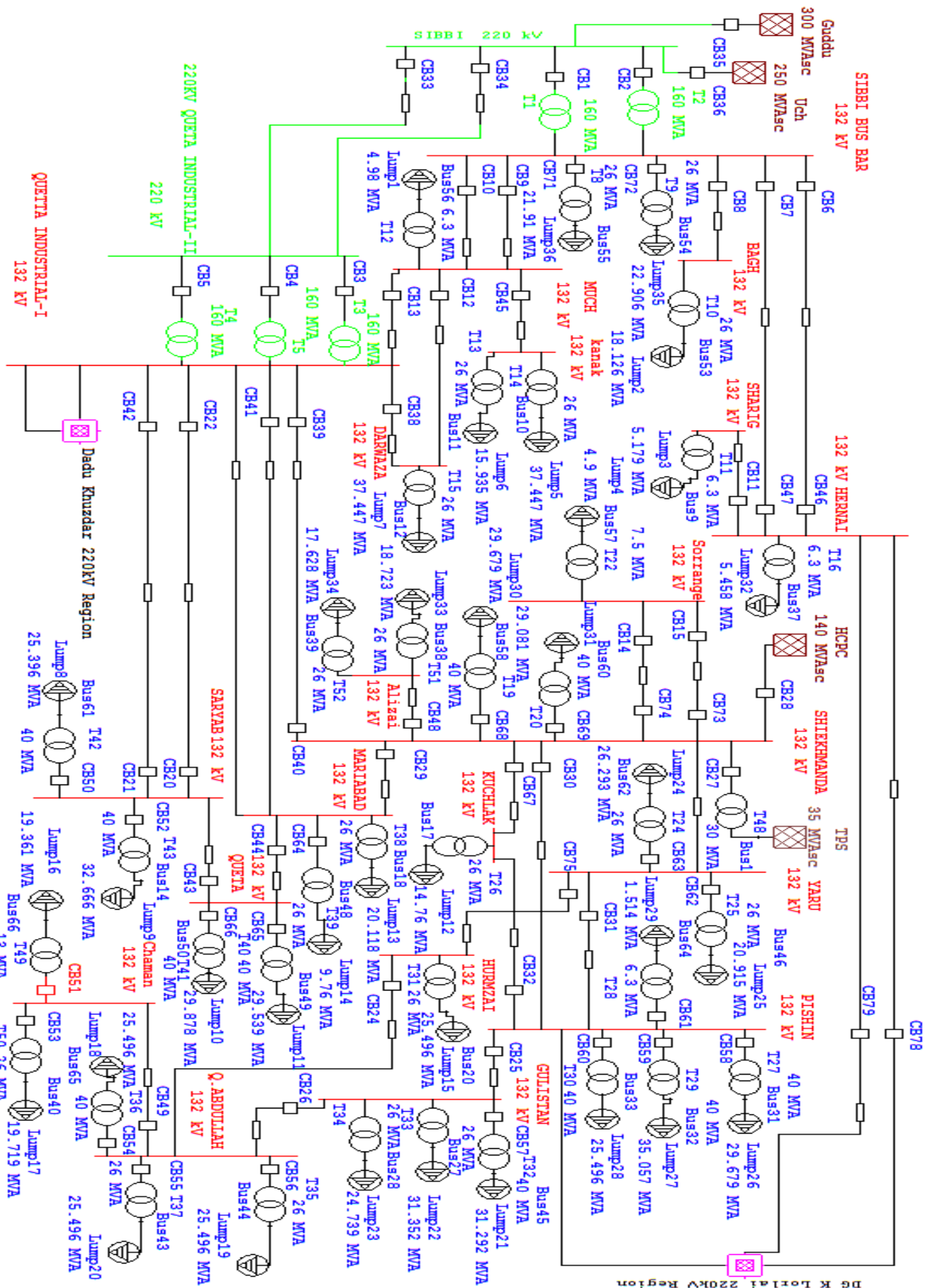

Figure 1: Maximum connected load of QESCO region (Single Line Diagram) 
The main objective of this research paper is to shed the non-preferred load of the QESCO network in the event of fault, in order to avoid complete power blackout of the company network. The 220kV transmission lines are connected with the National Grid in an inter-connected manner. To implement intelligent load shedding, Electrical Transient Analysis Program (ETAP) simulation tool is used. Load priority table containing the non- preferred (loads to be shed) is designed in ETAP as shown in Table- 3 . The load flow analysis is carried out for $\mathrm{N}-2$ contingency situations i.e. when both Daddu-Khuzdar and DG Khan-Loralai 220kV transmission lines are tripped.

Table 3: Load Priority Table

\begin{tabular}{|c|c|c|c|c|c|c|}
\hline S.No & $\begin{array}{c}\text { Name of Grid } \\
\text { Station }\end{array}$ & $\begin{array}{l}\text { Transformers } \\
\text { Connected }\end{array}$ & $\begin{array}{c}\text { Circuit } \\
\text { Breaker } \\
\text { Number }\end{array}$ & $\begin{array}{c}\text { Maximum } \\
\operatorname{Load}(\mathrm{A})\end{array}$ & $\begin{array}{l}\text { Maximum } \\
\text { Load } \\
\text { (MVA) }\end{array}$ & $\begin{array}{l}\text { Maximum } \\
\text { Load(MW) }\end{array}$ \\
\hline \multirow[b]{2}{*}{1} & \multirow[b]{2}{*}{ 132kv G/S Panjpai } & T-I (26 MVA) & CB138 & 700 & 14 & 11 \\
\hline & & T-II (13MVA) & CB138 & 440 & 8.8 & 7 \\
\hline \multirow[b]{2}{*}{2} & \multirow[b]{2}{*}{ 132kv G/S Kirdgab } & T-I (26 MVA) & CB137 & 1010 & 20 & 16 \\
\hline & & T-II (13MVA) & CB137 & 500 & 10 & 8 \\
\hline \multirow[b]{2}{*}{3} & \multirow[b]{2}{*}{ 132kv G/S Noshki } & T-I (26 MVA) & CB132 & 990 & 19.7 & 15 \\
\hline & & T-II (26 MVA) & CB132 & 1282 & 25.5 & 20.4 \\
\hline \multirow{3}{*}{5} & \multirow{3}{*}{ 132kv G/S Kharan } & T-I (26 MVA) & CB158 & 880 & 17.5 & 14 \\
\hline & & T-II (26 MVA) & CB158 & 820 & 16.3 & 13 \\
\hline & & T-III (26 VA) & CB158 & 1160 & 23 & 18.5 \\
\hline \multirow{3}{*}{6} & \multirow{3}{*}{$\begin{array}{c}132 \mathrm{kv} \text { G/S } \\
\text { Mangochar }\end{array}$} & T-I (40MVA) & CB155 & 1920 & 38.2 & 30.6 \\
\hline & & T-II (26 MVA) & CB153 & 1100 & 22 & 17.7 \\
\hline & & T-III (26 VA) & CB152 & 1120 & 22.3 & 17.8 \\
\hline \multirow[b]{2}{*}{7} & \multirow[b]{2}{*}{$132 \mathrm{kv}$ G/S Surab } & T-I (26 MVA) & CB128 & 940 & 18.7 & 15 \\
\hline & & T-II (13MVA) & CB129 & 650 & 13 & 10.4 \\
\hline \multirow[b]{2}{*}{8} & \multirow[b]{2}{*}{ 132kV G/S Gidder } & T-I (40 MVA) & CB120 & 1060 & 21 & 17 \\
\hline & & T-II (13MVA) & CB120 & 630 & 12.6 & 10 \\
\hline \multirow[b]{2}{*}{9} & \multirow[b]{2}{*}{$\begin{array}{c}\text { 132kV G/S Bagh } \\
\text { Bana }\end{array}$} & T-I (40 MVA) & $\begin{array}{l}\text { CB121- } \\
\text { CB122 }\end{array}$ & 1300 & 26 & 20.7 \\
\hline & & T-II (26 MVA) & $\begin{array}{l}\text { CB121- } \\
\text { CB122 }\end{array}$ & 590 & 11.8 & 9.4 \\
\hline \multirow[b]{2}{*}{10} & \multirow[b]{2}{*}{$132 \mathrm{kV} \mathrm{G} / \mathrm{S} \mathrm{Nal}$} & T-I (26 MVA) & CB118 & 1150 & 23 & 18 \\
\hline & & T-II (26 MVA) & CB118 & 1290 & 25.7 & 20.6 \\
\hline \multirow{3}{*}{11} & \multirow{3}{*}{ 132kV G/S Wadh } & T-I (26 MVA) & CB119 & 1126 & 22.4 & 18 \\
\hline & & T-II (13MVA) & CB119 & 617 & 12.3 & 9.8 \\
\hline & & T-III (13MVA) & CB119 & 390 & 7.8 & 6.2 \\
\hline \multirow{2}{*}{12} & \multirow{2}{*}{ 132kV G/S Zehri } & T-I (26 MVA) & CB154 & 1110 & 22 & 17.7 \\
\hline & & T-II (13MVA) & CB154 & 360 & 7.2 & 5.7 \\
\hline 13 & 132kV G/S Dalbandin & T-I (26 MVA) & CB131 & 150 & 3 & 2.4 \\
\hline 14 & 132kV G/S Mall & T-I (26 MVA) & CB131 & 1200 & 24 & 19 \\
\hline 15 & 132kV G/S Chaghi & T-I (13MVA) & CB130 & 40 & 0.8 & 0.6 \\
\hline 16 & 132kV G/S Baseema & T-I (13MVA) & CB160 & 221 & 4.4 & 3.5 \\
\hline 17 & 132kV G/S Makhter & T-I (13MVA) & CB90 & 393 & 7.8 & 6.2 \\
\hline 18 & 132kV G/S Kingri & T-I (6.3MVA & CB89 & 266 & 5.3 & 4.2 \\
\hline \multirow[t]{2}{*}{19} & \multirow[b]{2}{*}{ 132kV G/S Rakhni } & T-I (13MVA) & $\begin{array}{l}\text { CB98- } \\
\text { CB99 }\end{array}$ & 440 & 8.8 & 7 \\
\hline & & T-II (26MVA) & $\begin{array}{l}\text { CB98- } \\
\text { CB99 }\end{array}$ & 860 & 17 & 13.7 \\
\hline \multirow{2}{*}{20} & \multirow{2}{*}{ 132kV G/S Barkhan } & T-I (26MVA) & CB100 & 698 & 14 & 11 \\
\hline & & T-II (13MVA) & CB100 & 550 & 11 & 8.8 \\
\hline 21 & 132kV G/S Kohlu & T-I (13MVA) & CB101 & 601 & 12 & 9.6 \\
\hline
\end{tabular}




\begin{tabular}{|c|c|c|c|c|c|c|}
\hline \multirow[b]{2}{*}{22} & \multirow[b]{2}{*}{ 132kV G/S Zhob } & T-I (13MVA) & CB85 & 475 & 9.5 & 7.6 \\
\hline & & T-II (26MVA) & CB85 & 955 & 19 & 15.2 \\
\hline \multirow{2}{*}{23} & \multirow{2}{*}{ 132kV G/S M. Bagh } & T-I (26MVA) & CB81 & 1070 & 21.3 & 17 \\
\hline & & T-II (26MVA) & CB81 & 860 & 17 & 13.7 \\
\hline \multirow{2}{*}{24} & \multirow{2}{*}{ 132kV G/S G.H Zai } & T-I (26MVA) & CB83 & 1100 & 22 & 17.5 \\
\hline & & T-II (13MVA) & CB83 & 170 & 3.4 & 2.7 \\
\hline \multirow{2}{*}{25} & \multirow{2}{*}{ 132kV G/S M Pur } & T-I (6.3MVA) & CB84 & 230 & 4.6 & 3.7 \\
\hline & & T-II (26MVA) & CB84 & 430 & 8.6 & 6.8 \\
\hline \multirow{2}{*}{26} & \multirow{2}{*}{ 132kV G/S Khanozai } & T-I (26MVA) & CB102 & 1163 & 23 & 18.5 \\
\hline & & T-II (26MVA) & CB103 & 1194 & 24 & 19 \\
\hline 27 & 132kV G/S Ziarat & T-I (26MVA) & CB86 & 670 & 13.3 & 10.7 \\
\hline \multirow[t]{2}{*}{28} & 132kV G/S Dukki & T-I (13MVA) & $\begin{array}{l}\text { CB77- } \\
\text { CB79 }\end{array}$ & 990 & 19.7 & 15.8 \\
\hline & & T-II (13MVA) & $\begin{array}{l}\text { CB77- } \\
\text { CB79 } \\
\end{array}$ & 370 & 7.4 & 6 \\
\hline \multirow{3}{*}{29} & \multirow{3}{*}{$\begin{array}{l}\text { 132kV G/S } \\
\text { Q/Abdullah }\end{array}$} & T-I (40MVA) & $\begin{array}{l}\text { CB24- } \\
\text { CB26 }\end{array}$ & 1280 & 25.5 & 20.4 \\
\hline & & T-II (26MVA) & $\begin{array}{l}\text { CB24- } \\
\text { CB26 }\end{array}$ & 1280 & 25.5 & 20.4 \\
\hline & & T-III (26MVA) & $\begin{array}{l}\text { CB24- } \\
\text { CB26 }\end{array}$ & 1280 & 25.5 & 20.4 \\
\hline 30 & 132kV G/SSorrange & T-I (7.5MVA) & $\begin{array}{l}\text { CB14- } \\
\text { CB15 }\end{array}$ & 246 & 5 & 4 \\
\hline \multirow{2}{*}{31} & \multirow{2}{*}{ 132kV G/SAlizai } & T-I (26MVA) & CB48 & 940 & 18.7 & 15 \\
\hline & & T-II (26MVA) & CB48 & 885 & 17.6 & 14 \\
\hline 32 & 132kV G/SHuramzai & T-I (26MVA) & CB75 & 1280 & 25.5 & 20.4 \\
\hline \multirow[b]{2}{*}{33} & \multirow[b]{2}{*}{ 132kV G/SChaman } & T-I (13MVA) & CB49 & 972 & 19.4 & 15.5 \\
\hline & & T-II (26MVA) & CB49 & 990 & 19.7 & 15.8 \\
\hline 34 & 132kV G/SKuchlak & T-I (26MVA) & $\begin{array}{l}\text { CB67- } \\
\text { CB32 }\end{array}$ & 741 & 15 & 12 \\
\hline \multirow{3}{*}{35} & \multirow{3}{*}{ 132kV G/SGulistan } & T-I (40MVA) & $\begin{array}{l}\text { CB25- } \\
\text { CB26 }\end{array}$ & 1571 & 31.3 & 25 \\
\hline & & T-II (26MVA) & $\begin{array}{l}\text { CB25- } \\
\text { CB26 }\end{array}$ & 1574 & 31.4 & 25 \\
\hline & & T-III (26MVA) & $\begin{array}{l}\text { CB25- } \\
\text { CB26 }\end{array}$ & 1242 & 25 & 20 \\
\hline 36 & 132kV G/S Sharig & T-I (6.3MVA) & CB11 & 260 & 5.2 & 4 \\
\hline 37 & 132kV G/S Hernai & T-I (6.3MVA) & CB168 & 274 & 5.5 & 4.4 \\
\hline 38 & 132kV G/S Bagh & T-I (26MVA) & CB8 & 910 & 18 & 14.5 \\
\hline 39 & 132kV G/S Darwaza & T-I (26MVA) & $\begin{array}{l}\text { CB12- } \\
\text { CB38 }\end{array}$ & 1180 & 37 & 30 \\
\hline \multirow[b]{2}{*}{40} & \multirow[b]{2}{*}{ 132kV G/S Khanak } & T-I (26MVA) & CB45 & 1180 & 37 & 30 \\
\hline & & T-II (26MVA) & CB45 & 800 & 16 & 12.8 \\
\hline \multirow{3}{*}{41} & \multirow{3}{*}{ 132kV G/SMastung } & T-I (26 MVA) & CB144 & 1110 & 22 & 17.7 \\
\hline & & T-II (26 MVA) & CB143 & 906 & 18 & 14.4 \\
\hline & & T-III (26 VA) & CB144 & 920 & 18.3 & 14.7 \\
\hline \multirow{3}{*}{42} & & T-I (40MVA) & CB 183 & 995 & 19.8 & 15.8 \\
\hline & 132kV G/SQ Saifullah & T-II (40MVA) & CB 186 & 1030 & 20.5 & 16.4 \\
\hline & IJZKV G/SQ.Saliullan & T-III (40MVA) & CB 193 & 990 & 19.7 & 15.8 \\
\hline & & T-I (40 MVA) & CB150 & 1060 & 21 & 20 \\
\hline 43 & 132kV G/SKalat & T-II (26MVA) & CB126 & 630 & 12.6 & 15 \\
\hline & & T-I (26 MVA) & CB 136 & 1170 & 23.3 & 18.6 \\
\hline 44 & 132kV /SKhadKucha & T-II (26 MVA) & CB 145 & 1170 & 23.3 & 18.6 \\
\hline & TOTAL L & AD SHED & & 67097 & 1364 & 1091 \\
\hline
\end{tabular}




\section{RESULTS AND DISCUSSION}

The projected load demand and capacity of the three $220 \mathrm{kV}$ transmission lines of QESCO grid network is summarized in Table-4 (Marwat, 2017). It is clear from Table-4 that even in normal situation there is a shortage of $155 \mathrm{MW}$ in the network.

Table 4: Analysis of QESCO Network

\begin{tabular}{|c|c|c|c|c|}
\hline Network Topology & $\begin{array}{c}\text { Maximum } \\
\text { Demand (MW) }\end{array}$ & $\begin{array}{c}\text { Load } \\
\text { (MW) }\end{array}$ & $\begin{array}{c}\text { Losses } \\
\text { (MW) }\end{array}$ & Remarks \\
\hline Daddu- Khuzdar is tripped & 1961 & 1329 & 77 & $555 \mathrm{MW}$ needs to be shed \\
\hline Guddu-Sibbi is tripped & 1961 & 1244 & 176 & $541 \mathrm{MW}$ needs to be shed \\
\hline DG-khan Loralai is tripped & 1961 & 1292 & 133 & $536 \mathrm{MW}$ needs to be shed \\
\hline All 220 kV lines connected & 1961 & 1698 & 108 & $155 \mathrm{MW}$ needs to be shed \\
\hline
\end{tabular}

The load flow analysis after implementation of ILS in ETAP is shown in Table- 5 while the Single Line Diagram highlighting the active loads is given in Figure-2. The results of load flow analysis and SLD shows that with implementation of ILS the blackout of QESCO network could be avoided. The preferred load is still energized while the non-preferred load is shed as defined in load priority table.

Table 5: Load Flow of Energized Load of QESCO

\begin{tabular}{|c|c|c|c|c|c|c|}
\hline S. NO. & Name of grid station & $\begin{array}{l}\text { Transformers } \\
\text { Connected }\end{array}$ & Bus ID & $\begin{array}{c}\text { Maximum } \\
\operatorname{Load}(\mathrm{A})\end{array}$ & $\begin{array}{l}\text { Maximum } \\
\text { Load } \\
\text { (MVAR) }\end{array}$ & $\begin{array}{l}\text { Maximum } \\
\text { Load(MW) }\end{array}$ \\
\hline \multirow[b]{2}{*}{1} & \multirow[b]{2}{*}{ 132kV G/S Sariab } & T-I (40MVA) & 61 & 1275 & -15 & -20 \\
\hline & & T-II (40MVA) & 14 & 1640 & -19.6 & -26 \\
\hline \multirow[b]{2}{*}{2} & \multirow[b]{2}{*}{ 132kV G/S Marriabad } & T-I (26MVA) & 18 & 1010 & -12 & -16 \\
\hline & & T-II (26MVA) & 48 & 490 & -5.8 & -7.8 \\
\hline \multirow[b]{2}{*}{3} & \multirow[b]{2}{*}{$\begin{array}{c}\text { 132kV G/S Quetta } \\
\text { City }\end{array}$} & T-I (40MVA) & 49 & 1483 & -17.7 & -23.6 \\
\hline & & T-II (40MVA) & 50 & 1500 & -18 & -24 \\
\hline \multirow[b]{2}{*}{4} & \multirow[b]{2}{*}{$\begin{array}{c}132 k V \text { G/S } \\
\text { Shiekhmanda }\end{array}$} & T-I (40MVA) & 60 & 1460 & -17.4 & -23.3 \\
\hline & & T-II (40MVA) & 58 & 1490 & -17.8 & -24 \\
\hline \multirow{4}{*}{5} & \multirow{4}{*}{ 132kV G/S Pishin } & T-I (40MVA) & 31 & 1490 & -17.8 & -24 \\
\hline & & T-II (40MVA) & 32 & 1760 & -21 & -28 \\
\hline & & T-III (6.3MVA) & 64 & 76 & -0.9 & -1.2 \\
\hline & & T-IV (40MVA) & 33 & 1280 & -15.3 & -20.4 \\
\hline \multirow[b]{2}{*}{6} & \multirow[b]{2}{*}{ 132kV G/S Yaru } & T-I (26MVA) & 62 & 1320 & -15.8 & -21 \\
\hline & & T-II (26MVA) & 46 & 1050 & -12.5 & -16.7 \\
\hline 7 & 132kV G/S Hernai & T-I (6.3MVA) & 37 & 274 & -3.3 & -4.4 \\
\hline \multirow[b]{2}{*}{8} & \multirow[b]{2}{*}{ 132kV G/SSibbi } & T-I (26MVA) & 54 & 1150 & -13.4 & -18 \\
\hline & & T-II (26MVA) & 55 & 1100 & -13.2 & -17.5 \\
\hline \multirow{3}{*}{9} & \multirow{3}{*}{ 132kV G/SKhuzdar } & T-I (40 MVA) & 67 & 1570 & -18.8 & -25 \\
\hline & & T-II (18MVA) & 68 & 700 & -8.4 & -11 \\
\hline & & T-III (26 MVA) & 71 & 1060 & -12.7 & -17 \\
\hline 10 & 132kV G/SKalat & T-II (40 MVA) & 125 & 980 & -11.7 & -15.6 \\
\hline \multirow[b]{2}{*}{11} & \multirow[b]{2}{*}{ 132kV G/SLoralai } & T-I (26MVA) & 157 & 1096 & -13.1 & -17.5 \\
\hline & & T-II (40MVA) & 156 & 1885 & -22.5 & -30 \\
\hline \multirow{3}{*}{12} & \multirow{3}{*}{ 132kV G/SQ/Saifullah } & T-I (40MVA) & 165 & 995 & -11.9 & -15.8 \\
\hline & & T-II (40MVA) & 166 & 1030 & -12.3 & -16.4 \\
\hline & & T-III (40MVA) & 164 & 990 & -11.8 & -15.8 \\
\hline \multirow[b]{2}{*}{13} & \multirow[b]{2}{*}{ 132kV G/S M. Bagh } & T-I (26MVA) & 167 & 1070 & -12.8 & -17 \\
\hline & & T-II (26MVA) & 168 & 860 & -10.3 & -13.7 \\
\hline \multicolumn{4}{|c|}{ TOTAL LOAD ENERGIZED } & 32084 & -382.8 & -510.7 \\
\hline
\end{tabular}


Blackout Avoidance through Intelligent Load Shedding in Modern Electrical Power Utility Network

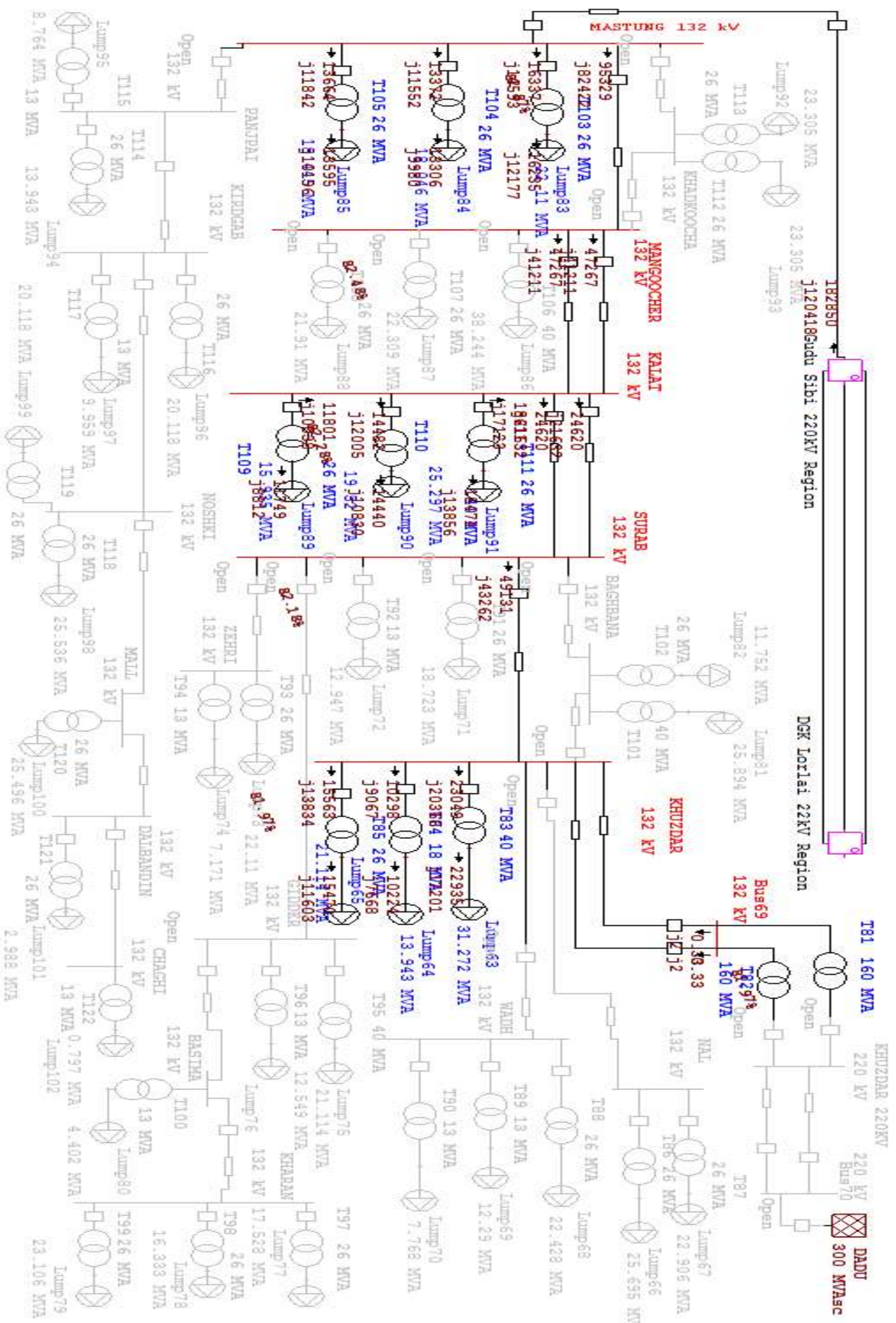

Figure 2: Load Flow of Energized Load of QESCO 
As, we were deficient of combined input power of both Daddu-Khuzdar and D.G Khan-Loralai (220kV lines), we disconnected 1091 MW of load through Load Priority Table in Table-3.

\section{CONCLUSION}

The maintenance and sustained operation of electrical power system is complex and cumbersome job. In Pakistan, it is more challenging to prevent the National Grid from blackouts and maintain the electrical power system stability. To avoid power system blackout, in this research work an idea of Automatic Node-Switching or Intelligent Load Shedding (ILS) system is introduced. Through ILS non-preferred load of a power system has been disconnected to avoid the tripping of whole power utility network. For simulating ILS, Electrical Transient Analysis Program (ETAP) software was used. The Automatic Node-Switching operation of ETAP Real-Time is based on maintaining system stability with minimum load shed. The Automatic Node-Switching has been applied at the 220kV transmission network of QESCO through different scenarios. The technique of ILS, if applied, is helpful in maintaining the stability and power security of National Grid or any one of the Distribution Companies (DISCOS). Major breakdowns and calamities of the power system can be easily handled without collapsing the entire electrical power system of the utility company. This will lessen the losses and damage of the expensive power equipment from power collapses.

\section{REFERENCES}

- Adibi M. (2015). IEEE Life Fellow, Nelson Martins IEEE Fellow. Impact of Power System Blackouts, IEEE Power \& Energy Society General Meeting, USA.

- Złotecka D, Sroka K. (2018). The characteristics and main causes of power system failures basing on the analysis of previous blackouts in the world, 2018 International Interdisciplinary PhD Workshop (IIPhDW), Świnoujście, Poland.

- Wang F, Li C, Li L. (2018). Fractal Characteristics Analysis of Blackouts in Interconnected Power Grid. IEEE Transactions on Power Systems 33(1):1-3.

- McLellzm M. (1998). Influence of power system protection on system blackouts, IEEE Colloquium on Measures to Prevent Power Blackouts (Digest No. 1998 /292), England.

- Fong E, Chiu DKW, Hu H, Zhuang Y, Hu H. (2011). Blackout Avoidance and Energy Saving with Alert Aggregation, 5th International Conference on New Trends in Information Science and Service Science (NISS), China.

- Babalola AA, Belkacemi R. (2018). Real-Time Cascading Failures Prevention for Multiple Contingencies in Smart Grids Through a Multi-Agent System. IEEE Transactions on Smart Grid. 9(1):373-385.

- Younas MW, Qureshi SA. (2006). Analysis of Blackout of National Grid System of Pakistan in 2006 and the Application of PSS and FACTS Controllers as Remedial Measures, Pakistan.

- Bashir N, Sharani Z, Qayyum K, Syed AA. (2015). Delivering Smart Load-shedding for Highly-stressed Grids, IEEE International Conference on Smart Grid Communications (Smart Grid Comm): Architectures, Control and Operation for Smart Grids and Micro grids.

- Kafait-Ullah. (2013). Electricity Infrastructure in Pakistan: An Overview. International Journal of Energy, Information and Communications 4(3):11-26.

- Scutariu M, MacDonald M. (2009). Industrial Power System Protection against Transmission System Blackouts, 44th International Universities Power Engineering Conference (UPEC), Glasgow, UK.

- Yagami M, Ichinohe Y. (2017). Transient Stability Analysis of Synchronous Generator in Power System with Renewable Power Sources Installed, 19th International Conference on Electrical Machines and Systems, Chiba Japan.

- Marwat I. (2017). Avoidance of Blackouts using Automatic Node-switching Technique through ETAP. International Journal of Scientific \& Engineering Research 8(10): 715-720. 